Archive for

Organic Chemistry

Arkivoc 2018, part v, 0-0

\title{
New $C_{2}$-symmetric six-membered carbene ligands for asymmetric diethylzinc addition of arylaldehydes
}

\author{
Bihui Zhou, ${ }^{\text {a,b }}$ Zijing Li, ${ }^{\text {a }}$ Chen Yang, ${ }^{a}$ Linlin Li, ${ }^{\text {a Limei Fan, }}{ }^{\text {a Hongxia Huang, }}{ }^{\text {a }}$ and Jie Li*a \\ ${ }^{a}$ Department of Pharmacy, School of Medicine, Zhejiang University City College, No. 48, Huzhou Road, \\ Hangzhou 310015, PR China \\ ${ }^{b}$ College of Pharmaceutical Siences, Zhejiang University, Hangzhou 310058, PR China \\ Email: lijie@zucc.edu.cn
}

Received 11-25-2017

Accepted 02-17-2018

Published on line 05-28-2018

\section{Abstract}

A series of new six-membered NHC precursors were prepared by simply esterification of their parent compounds. Their applicability in asymmetric diethylzinc addition of arylaldehydes has been demonstrated and the corresponding secondary alcohol was obtained with good yields and moderate enantioselectivities.

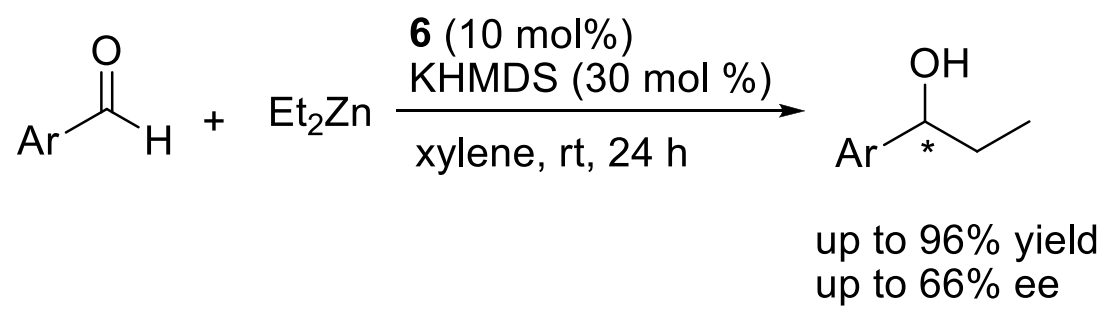

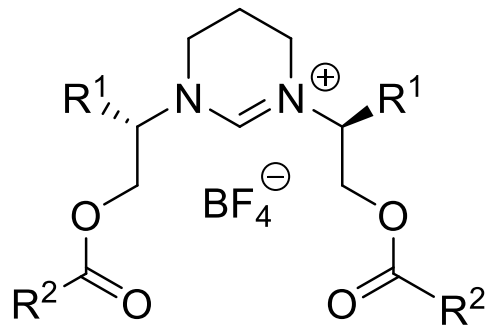

6

Keywords: N-Heterocyclic carbene, tetrahydropyrimidinium, amino alcohols, asymmetric diethylzinc addition, chiral secondary alcohols 


\section{Introduction}

Since the first isolation of free N-heterocyclic carbene (NHC) by Arduengo's group in $1991,{ }^{1}$ these types of ligands have gathered considerable interests because of their attractive properties such as higher stability to air, thermal and moisture than phosphane ligands, and NHCs are now ubiquitous in modern synthetic chemistry. ${ }^{2-12}$ As an extension, the development of new chiral NHC precursors for catalysis has become an important issue and an enormous number of new chiral carbenes emerged, some of which have shown excellent enantioselectivity in asymmetric catalysis. ${ }^{13-18}$ However, among the chiral NHCs synthesized, examples of chiral hydroxyalkyl NHC ligands are still rare. In 2004, Arnold and co-workers reported the synthesis of salt 1 (Figure 1), ${ }^{19}$ the $\mathrm{Cu}^{\prime}$ complex of this ligand was used as a catalyst in diethylzinc conjugated addition to cyclohexenone, affording the desired product in up to $51 \%$ ee. Almost at the same time, another type of hydroxy-bearing NHC salt $\mathbf{2}$ derived from (L)-valine was prepared by Mauduit's group and this compound showed high efficiency in chiral molecular recognition. ${ }^{20}$ Furthermore, the same group designed and synthesized a series of new bidentate NHC precursors 3 based on commercial available amino alcohols, ${ }^{21-}$

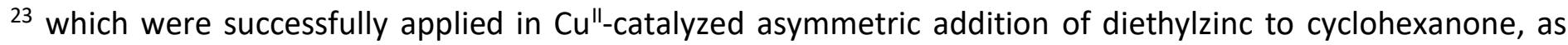
well as asymmetric allylic substitution of allyl phosphates with Grignard reagents. Moreover, the same ligand $\mathbf{3}$ was proved to be an excellent ligand in multicomponent catalytic enantioselective transformations. ${ }^{24}$ With similar starting materials, Wilhelm's group prepared several new tridentate NHC precursors 4 as ionic liquids, ${ }^{25-26}$ and these salts were also used as catalyst in asymmetric diethylzinc addition to arylaldehydes, giving the corresponding secondary alcohols in good yields and moderate ees (up to 66\%).

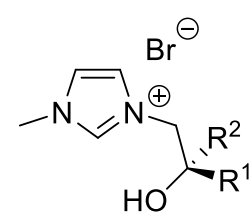

1

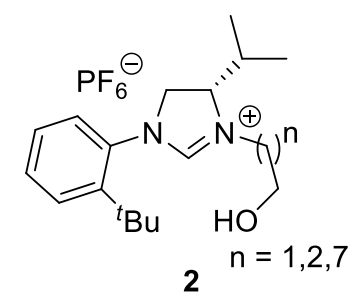

2

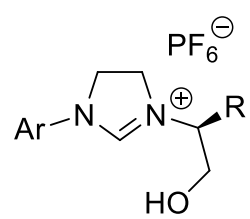

3
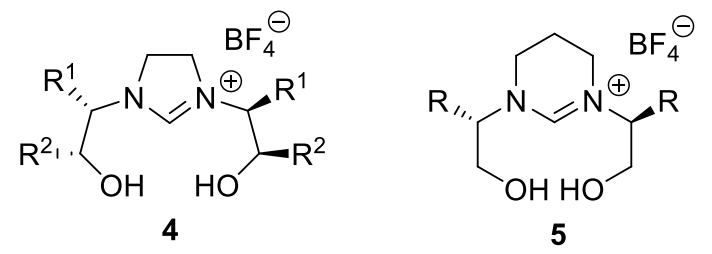

Figure 1. Representative chiral hydroxyalkyl NHC ligands.

\section{Results and Discussion}

Very recently, we reported the synthesis of several enantiopure 3,4,5,6-tetrahydropyrimidinium salts $\mathbf{5}$ incorporating two hydroxyl groups as effective ligands in Pd-catalyzed deprotonative cross-coupling process (DCCP). ${ }^{27}$ Next, we examined the activity of these ligands in asymmetric diethylzinc addition to aldehydes. The yields are good but the ee values are rather low (Table 1, entries 1-6). Usually, the hydroxy group in the Nsubstituent of the NHC ligand was introduced as a coordination group to block the rotation of $N$-substituent which subsequently elevates the enantioselectivity in catalytic transformations. On the other hand, little 
attention was paid to modifications the $\mathrm{OH}$ group with a steric functional group, which may result in an elevated chiral environment around the carbene center. With this expectation in mind, a series of derivatives of salts $\mathbf{5}$ are therefore prepared in this paper with the aim to improve their performance in asymmetric catalysis. As shown in Scheme 1, simple treatment of compounds 5a-f with 4-(tert-butyl)benzoyl chloride provided esterification product $6 a-f$ in good yields (76-92\%). Luckily, single crystals of $6 f$ were obtained from $\mathrm{CH}_{2} \mathrm{Cl}_{2}$, and the ORTEP view of this compound was obtained (Figure 2).

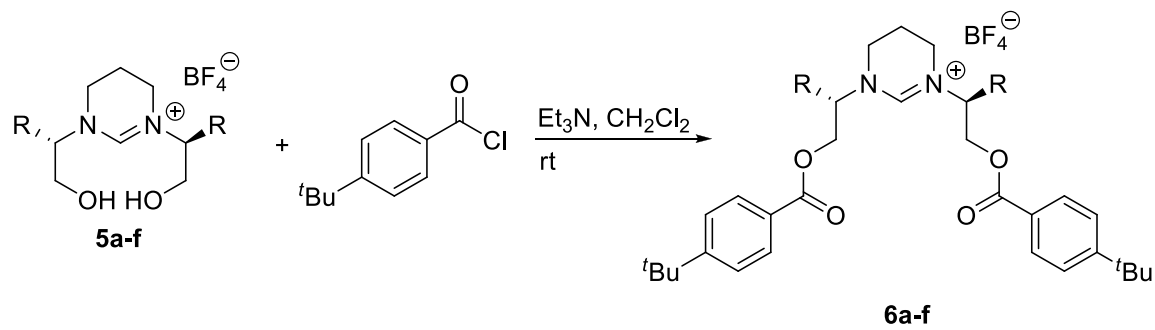<smiles>CCC(C)[C@@H](COC(=O)c1ccc(Br)cc1)N1CCCN([C@@H](COC(=O)c2ccc(Br)cc2)C(C)CC)C1</smiles>

$6 a$

6b

$6 \mathrm{c}$

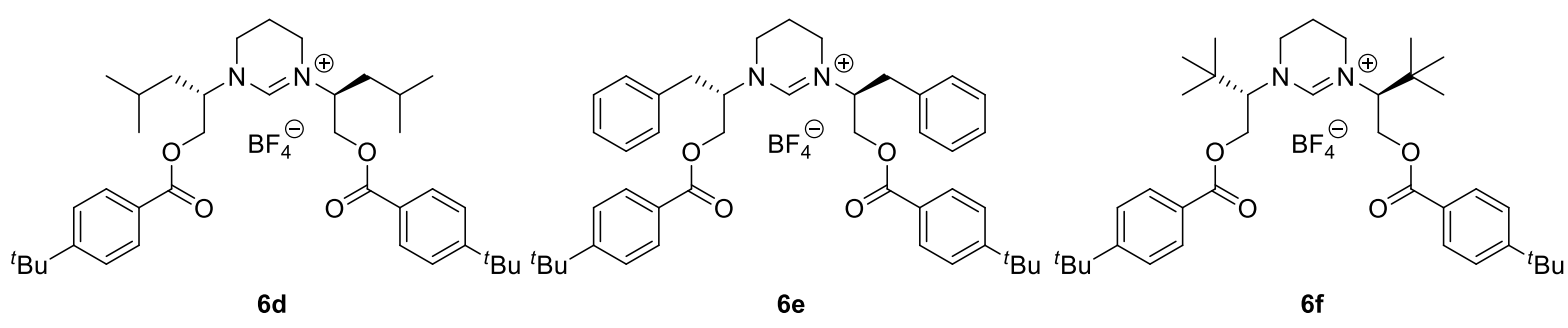

Scheme 1. Synthesis of NHC precursors $6 \mathbf{a}-\mathbf{f}$.

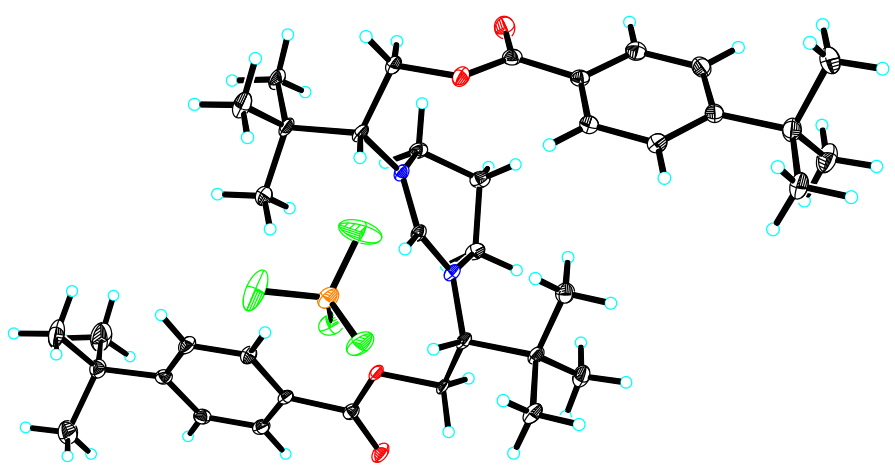

Figure 2. Crystal structure of $\mathbf{6 f}$. 
Table 1. Comparison of NHC precursors

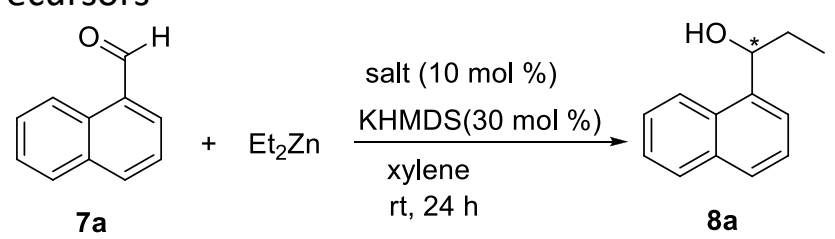

\begin{tabular}{cccc}
\hline Entry $^{\text {a }}$ & Catalyst & ${\text { Yield }(\%)^{\text {b }}}$ & ee $(\%)^{\mathrm{c}}$ \\
\hline 1 & $\mathbf{5 a}$ & 89 & 4 \\
2 & $\mathbf{5 b}$ & 92 & 21 \\
3 & $\mathbf{5 c}$ & 81 & 9 \\
4 & $\mathbf{5 d}$ & 90 & 5 \\
5 & $\mathbf{5 e}$ & 86 & 13 \\
6 & $\mathbf{5 f}$ & 77 & 1 \\
7 & $\mathbf{6 a}$ & 73 & 7 \\
8 & $\mathbf{6 b}$ & 93 & 58 \\
9 & $\mathbf{6 c}$ & 80 & 20 \\
10 & $\mathbf{6 d}$ & 83 & 8 \\
11 & $\mathbf{6 e}$ & 81 & 41 \\
12 & $\mathbf{6 f}$ & 95 & 7 \\
13 & $\mathbf{6 g}$ & 90 & 9 \\
14 & $\mathbf{6 h}$ & 83 & 11 \\
15 & $\mathbf{6 i}$ & 92 & 23 \\
\hline
\end{tabular}

${ }^{a}$ Reaction condition: salt (10 mol \%), KHMDS (30 mol \%), Et $2 \mathrm{Zn}$ (2 equiv), $\mathrm{N}_{2}$, xylene, rt, 24 h. ${ }^{b}$ Isolated yield. ${ }^{c}$ Determined by chiral HPLC (CHIRALCEL OD Column) analysis.

The synthesized tetrahydropyrimidinium salts 6 were tested in asymmetric diethylzinc addition of 1naphthaldehyde (7a) according to our procedure in the same transformation with pyrimidone salts as catalysts. ${ }^{28}$ As presented in Table 1, all derivatives 6a-f showed better enantioselectivities than their parent compounds $\mathbf{5 a - f}$, and $\mathbf{6} \mathbf{b}$ gave the best result ( $93 \%$ yield, $58 \%$ ee). We then tried various conditions of different bases and solvents. Unfortunately, all combinations didn't improve the enantioselectivity (see Supporting information for details). Futhermore, three new salts 6g-6i (Figure 3), derived from the same parent compound as $\mathbf{6 b}$, were also prepared and tested in the same reaction, no improvement of ee value was observed as well (entries 13-15).
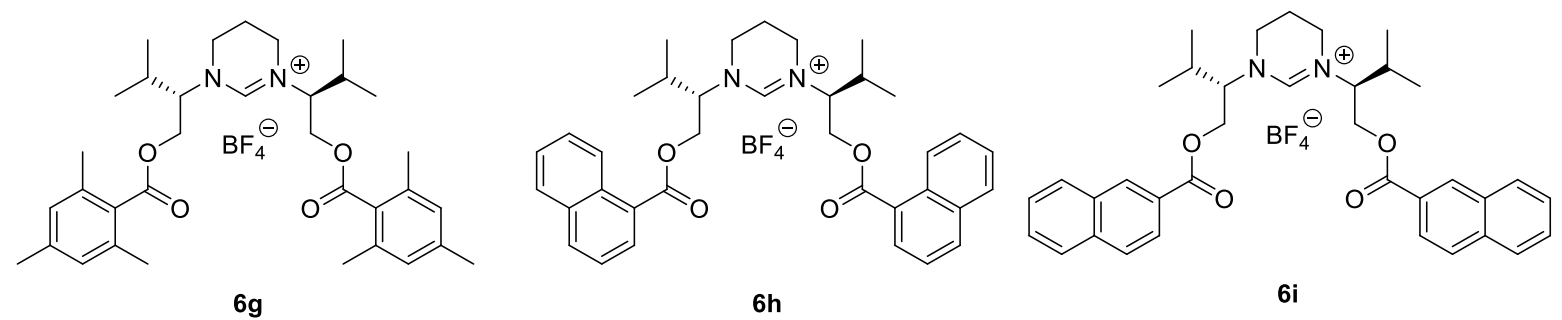

6h

$6 \mathbf{i}$

Figure 3. The structures of $\mathbf{6 g - 6 i}$.

Using $\mathbf{6 b}$ as catalyst, various arylaldehydes with different substituents were examined in this transformation. As summarized in Table 2, the reaction proceeded well in most cases (73-96\% yield). Arylaldehydes bearing electron-donating (entries 3-7) and electron-withdrawing (entries 8-11) groups, as well 
as heterocyclic sustrates (entries 13-15), were all well-tolerated, giving the corresponding adducts $\mathbf{8 b - 8} \mathbf{p}$ in good yields and moderate enantiomeric excesses. The best enantioselectivity was obtained starting from 2 quinolinecarbaldehyde (66\% ee, entry 15$)$.

Table 2. Scope of methodology

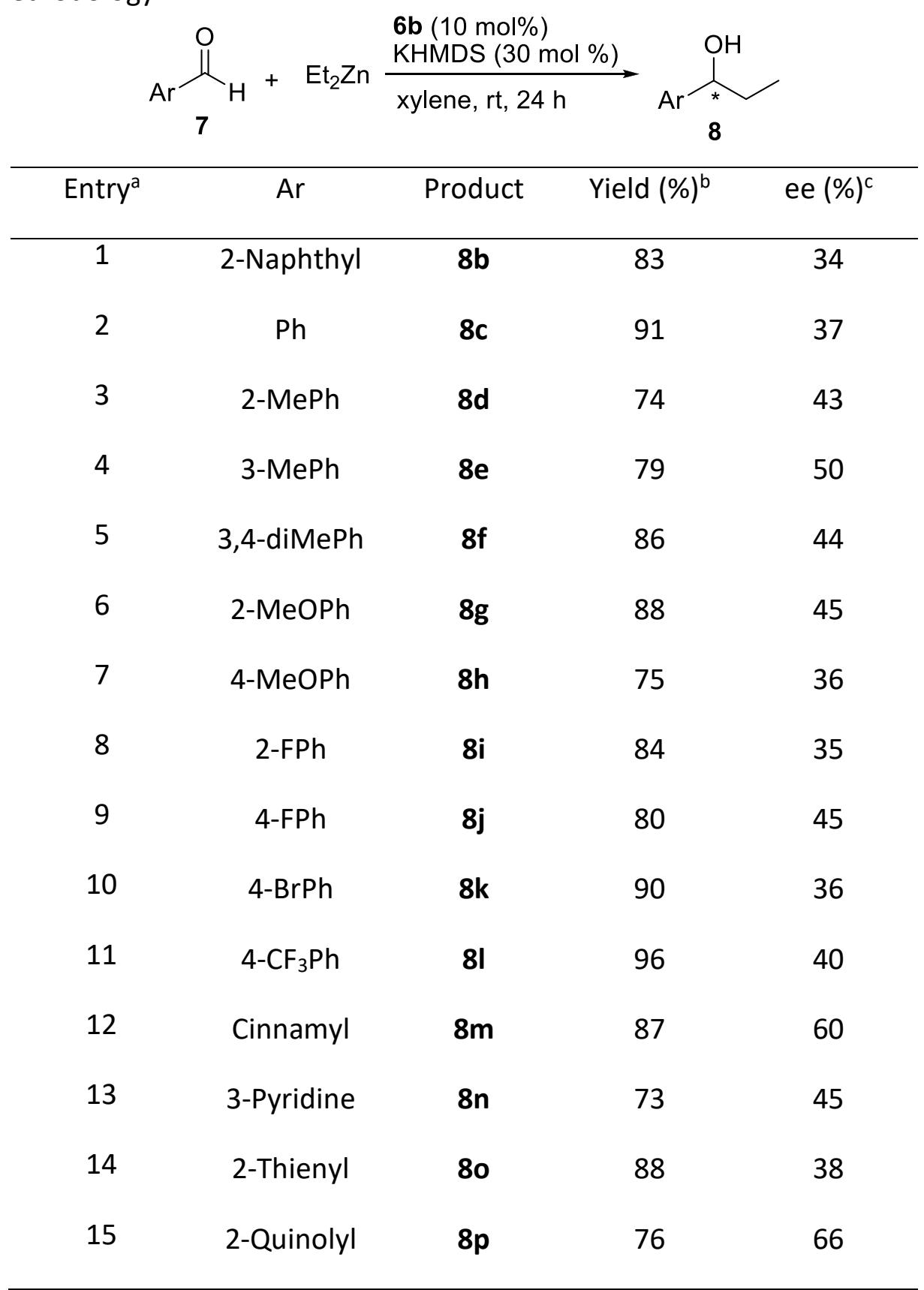

a Reaction condition: $6 \mathbf{b}$ (10 mol \%), KHMDS (30 mol \%), Et $2 \mathrm{Zn}$ (2 equiv), N2, xylene, rt, 24 h.

${ }^{b}$ Isolated yields. ${ }^{c}$ Determined by chiral HPLC (CHIRALCEL OD Column) analysis.

\section{Conclusions}

In summary, a series of new six-membered NHC precursors (6a-6i) have been prepared and the single-crystal X-ray diffraction further confirmed the structure of compound $\mathbf{6 f}$. The catalytical activity of these ligands in 
asymmetric diethylzinc addition to arylaldehydes was tested and the corresponding secondary alcohols were obtained with excellent yields and moderate ees (up to 66\%). Further work is currently underway to prepare more chiral six-membered NHC ligands, as catalysts in other asymmetric transformation, by modification the hydroxyl group in $N$-substituent.

\section{Experimental Section}

General. MS spectra were measured on a Finnigan LCQDECA XP instrument and a Agilent Q-TOF 1290 LC/6224 MS system; ${ }^{1} \mathrm{H}$ and ${ }^{13} \mathrm{C}$ NMR spectra were obtained on Bruker AVANCE III $500 \mathrm{MHz}$ and $600 \mathrm{MHz}$ spectrometers (Bruker Co., Switzerland) with TMS as the internal standard; silica gel GF 254 and H (10-40 mm, Qingdao Marine Chemical Factory, China) were used for TLC and CC. Unless otherwise noted, all reactions were carried out under an atmosphere of argon or nitrogen.

General procedure for the synthesis of compounds 6 . To a mixture of salt 5 ( $2 \mathrm{mmol}$ ) and $\mathrm{Et}_{3} \mathrm{~N}(9.6 \mathrm{mmol})$ in dry dichloromethane $(10 \mathrm{~mL})$ was added 4-tert-butylbenzoyl chloride $(8 \mathrm{mmol})$ at $0{ }^{\circ} \mathrm{C}$. After stiring at room temperature for $12 \mathrm{~h}$, the mixture was poured into water $(25 \mathrm{~mL})$ and extracted with dichloromethane $(3 \times 10$ $\mathrm{mL}$ ). The organic fractions were combined, washed with brine and dried over $\mathrm{Na}_{2} \mathrm{SO}_{4}$. The solvent was then removed under reduced pressure and the residue was purified by column chromatography (silica gel, $\mathrm{CH}_{2} \mathrm{Cl}_{2} / \mathrm{MeOH}$ ) to afford the corresponding products 6 .

6a. White powder; yield $1.31 \mathrm{~g}(89 \%) ;{ }^{1} \mathrm{H}$ NMR $\left(500 \mathrm{MHz}, \mathrm{CDCl}_{3}\right) \delta 9.22(\mathrm{~s}, 1 \mathrm{H}), 8.19(\mathrm{~d}, J 8.6 \mathrm{~Hz}, 4 \mathrm{H}), 7.60(\mathrm{~d}, J$ 8.6 Hz, 4H), 7.29 (d, J $7.5 \mathrm{~Hz}, 2 \mathrm{H}), 7.13(\mathrm{t}, J 7.8 \mathrm{~Hz}, 4 \mathrm{H}), 6.99(\mathrm{~d}, J 7.5 \mathrm{~Hz}, 4 \mathrm{H}), 5.61(\mathrm{dd}, J 10.7,3.5 \mathrm{~Hz}, 2 \mathrm{H}), 5.46-$ $5.35(\mathrm{~m}, 2 \mathrm{H}), 4.47(\mathrm{~m}, 2 \mathrm{H}), 3.35-3.21(\mathrm{~m}, 2 \mathrm{H}), 2.89-2.76(\mathrm{~m}, 2 \mathrm{H}), 1.80-1.73(\mathrm{~m}, 2 \mathrm{H}), 1.38(\mathrm{~s}, 18 \mathrm{H}) ;{ }^{13} \mathrm{C} \mathrm{NMR}$ $\left(125 \mathrm{MHz}_{\mathrm{CDCl}}\right) \delta 166.44,157.70,131.59,131.58,130.14,129.48,129.32,127.57,125.95,125.85,65.75$, 60.10, 38.75, 35.27, 31.16, 18.65; ESIMS $m / z$ 645.3.

6b. $81 \%$ yield; ${ }^{1} \mathrm{H}$ NMR $\left(500 \mathrm{MHz}, \mathrm{CDCl}_{3}\right) \delta 8.53(\mathrm{~s}, 1 \mathrm{H}), 7.99(\mathrm{~d}, J 8.6 \mathrm{~Hz}, 4 \mathrm{H}), 7.46(\mathrm{~d}, J 8.6 \mathrm{~Hz}, 4 \mathrm{H}), 4.78(\mathrm{dd}, J$ 12.4, $10.4 \mathrm{~Hz}, 2 \mathrm{H}), 4.27$ (dd, J 12.4, $3.4 \mathrm{~Hz}, 2 \mathrm{H}), 3.95(\mathrm{~m}, 2 \mathrm{H}), 3.47-3.37(\mathrm{~m}, 2 \mathrm{H}), 3.20-3.09(\mathrm{~m}, 2 \mathrm{H}), 2.03-$ $1.94(\mathrm{~m}, 2 \mathrm{H}), 1.82(\mathrm{dd}, J 6.5,4.0 \mathrm{~Hz}, 2 \mathrm{H}), 1.33(\mathrm{~s}, 18 \mathrm{H}), 1.02(\mathrm{~d}, J 6.6 \mathrm{~Hz}, 6 \mathrm{H}), 0.52(\mathrm{~d}, J 6.6 \mathrm{~Hz}, 6 \mathrm{H}) ;{ }^{13} \mathrm{C} \mathrm{NMR}$ $\left(125 \mathrm{MHz}_{1} \mathrm{CDCl}_{3}\right) \delta 166.35,157.56,155.43,129.90,125.88,125.71,70.31,60.73,38.82,35.17,31.06,26.80$, 19.10, 18.98; HR-ESIMS: $m / z 578.4090\left[\mathrm{M}-\mathrm{BF}_{4}+\mathrm{H}\right]^{+}$(calcd for $\mathrm{C}_{36} \mathrm{H}_{54} \mathrm{~N}_{2} \mathrm{O}_{4}{ }^{+}, 578.4078$ ).

6c. $84 \%$ yield; ${ }^{1} \mathrm{H}$ NMR $\left(500 \mathrm{MHz}, \mathrm{CDCl}_{3}\right) \delta 8.53(\mathrm{~s}, 1 \mathrm{H}), 8.02(\mathrm{~d}, J 8.6 \mathrm{~Hz}, 4 \mathrm{H}), 7.49(\mathrm{~d}, J 8.7 \mathrm{~Hz}, 4 \mathrm{H}), 4.80(\mathrm{dd}, J$ 12.3, 10.5 Hz, 2H), 4.25 (dd, J 12.4, 3.4 Hz, 2H), $4.03(\mathrm{~m}, 2 \mathrm{H}), 3.43(\mathrm{dd}, J 12.7,6.2 \mathrm{~Hz}, 2 \mathrm{H}), 3.16-3.07(\mathrm{~m}, 2 \mathrm{H})$, $2.02-1.98(\mathrm{~m}, 2 \mathrm{H}), 1.57-1.52(\mathrm{~m}, 2 \mathrm{H}), 1.33(\mathrm{~s}, 18 \mathrm{H}), 0.97(\mathrm{~d}, J 6.6 \mathrm{~Hz}, 6 \mathrm{H}), 0.87-0.81(\mathrm{~m}, 2 \mathrm{H}), 0.75(\mathrm{~m}, 2 \mathrm{H})$, $0.50(\mathrm{t}, \mathrm{J} 7.4 \mathrm{~Hz}, 6 \mathrm{H}) ;{ }^{13} \mathrm{C} \mathrm{NMR}\left(125 \mathrm{MHz} \mathrm{CDCl}_{3}\right) \delta 166.37,157.42,155.34,129.82,126.01,125.72,69.17$, 61.05, 39.06, 35.14, 32.99, 31.05, 25.10, 18.74, 14.98, 10.69; HR-ESIMS: $\mathrm{m} / z$ 605.4395 [M-BF $\left._{4}\right]^{+}$(calcd for $\left.\mathrm{C}_{38} \mathrm{H}_{57} \mathrm{~N}_{2} \mathrm{O}_{4}{ }^{+}, 605.4313\right)$.

6d. $92 \%$ yield; ${ }^{1} \mathrm{H}$ NMR $\left(500 \mathrm{MHz}, \mathrm{CDCl}_{3}\right) \delta 8.53(\mathrm{~s}, 1 \mathrm{H}), 8.02(\mathrm{~d}, J 8.6 \mathrm{~Hz}, 4 \mathrm{H}), 7.49(\mathrm{~d}, J 8.6 \mathrm{~Hz}, 4 \mathrm{H}), 4.75(\mathrm{~m}$, $2 \mathrm{H}), 4.35$ (dd, J 9.3, $4.6 \mathrm{~Hz}, 2 \mathrm{H}$ ), $4.02(\mathrm{dd}, J 12.4,3.4 \mathrm{~Hz}, 2 \mathrm{H}), 3.53-3.42(\mathrm{~m}, 2 \mathrm{H}), 3.27-3.16(\mathrm{~m}, 2 \mathrm{H}), 2.05(\mathrm{dd}, J$ 7.3, $4.2 \mathrm{~Hz}, 2 \mathrm{H}), 1.43(\mathrm{~m}, 2 \mathrm{H}), 1.33(\mathrm{~s}, 18 \mathrm{H}), 1.31-1.27(\mathrm{~m}, 2 \mathrm{H}), 1.17-1.10(\mathrm{~m}, 2 \mathrm{H}), 0.72(\mathrm{~d}, J 6.6 \mathrm{~Hz}, 6 \mathrm{H}), 0.63$ $(\mathrm{d}, J 6.5 \mathrm{~Hz}, 6 \mathrm{H}) ;{ }^{13} \mathrm{C} \mathrm{NMR}\left(125 \mathrm{MHz}, \mathrm{CDCl}_{3}\right) \delta 166.46,157.52,154.79,129.92,125.89,125.77,62.60,62.31$, $38.47,36.24,35.18,31.08,29.70,24.61,22.82,21.46$; HR-ESIMS: $\mathrm{m} / z$ 605.4410 [M-BF $]^{+}$(calcd for $\left.\mathrm{C}_{38} \mathrm{H}_{57} \mathrm{~N}_{2} \mathrm{O}_{4}{ }^{+}, 605.4313\right)$.

6e. $85 \%$ yield; ${ }^{1} \mathrm{H}$ NMR $\left(500 \mathrm{MHz}, \mathrm{CDCl}_{3}\right) \delta 8.60(\mathrm{~s}, 1 \mathrm{H}), 7.96-7.89(\mathrm{~m}, 4 \mathrm{H}), 7.46-7.41(\mathrm{~m}, 4 \mathrm{H}), 7.25-7.13(\mathrm{~m}$, $10 \mathrm{H}), 4.68(\mathrm{dd}, m, 2 \mathrm{H}), 4.54(\mathrm{~d}, J 9.8 \mathrm{~Hz}, 2 \mathrm{H}), 4.24(\mathrm{dd}, J 12.4,3.5 \mathrm{~Hz}, 2 \mathrm{H}), 3.14(\mathrm{~m}, 4 \mathrm{H}), 2.99(\mathrm{dd}, J \mathrm{~m}, 2 \mathrm{H}), 2.79$ 
$(\mathrm{m}, 2 \mathrm{H}), 1.74-1.66(\mathrm{~m}, 2 \mathrm{H}), 1.25(\mathrm{~s}, 18 \mathrm{H}) ;{ }^{13} \mathrm{C}$ NMR $\left(125 \mathrm{MHz}, \mathrm{CDCl}_{3}\right) \delta 166.04,157.52,154.32,134.83$, $129.71,129.11,128.88,127.42,125.99,125.72,65.24,62.84,41.03,35.11,34.99,30.98,18.66$; HR-ESIMS: $m / z$ $673.4094\left[\mathrm{M}-\mathrm{BF}_{4}\right]^{+}$(calcd for $\mathrm{C}_{44} \mathrm{H}_{53} \mathrm{~N}_{2} \mathrm{O}_{4}{ }^{+}, 673.4000$ ).

6f. $76 \%$ yield; ${ }^{1} \mathrm{H}$ NMR (500 MHz, $\left.\mathrm{CDCl}_{3}\right) \delta 8.58(\mathrm{~s}, 1 \mathrm{H}), 7.99(\mathrm{~d}, J 7.3 \mathrm{~Hz}, 4 \mathrm{H}), 7.45(\mathrm{~d}, J 8.1 \mathrm{~Hz}, 4 \mathrm{H}), 4.98(\mathrm{t}, J$ $11.5 \mathrm{~Hz}, 2 \mathrm{H}), 4.23(\mathrm{~m}, 4 \mathrm{H}), 3.50(\mathrm{dd}, J 12.8,6.1 \mathrm{~Hz}, 2 \mathrm{H}), 3.35-3.26(\mathrm{~m}, 2 \mathrm{H}), 2.01-1.93(\mathrm{~m}, 2 \mathrm{H}), 1.33(\mathrm{~s}, 18 \mathrm{H})$, $1.26(\mathrm{~s}, 18 \mathrm{H}) ;{ }^{13} \mathrm{C} \mathrm{NMR}\left(125 \mathrm{MHz}, \mathrm{CDCl}_{3}\right) \delta 166.32,157.36,129.81,126.03,125.56,72.41,58.69,40.65,35.11$, 33.98, 31.05, 29.67, 27.37, 18.81; HR-ESIMS: $m / z$ 605.4412 [M-BF $]^{+}$(calcd for $\mathrm{C}_{38} \mathrm{H}_{57} \mathrm{~N}_{2} \mathrm{O}_{4}{ }^{+}, 605.4313$ ).

6g. 84\% yield; ${ }^{1} \mathrm{H}$ NMR $\left(500 \mathrm{MHz}, \mathrm{CDCl}_{3}\right) \delta 8.40(\mathrm{~s}, 1 \mathrm{H}), 6.83(\mathrm{~s}, 4 \mathrm{H}), 4.52(\mathrm{~m}, 4 \mathrm{H}), 3.89-3.80(\mathrm{~m}, 2 \mathrm{H}), 3.38(\mathrm{dd}$, J 12.8, $6.5 \mathrm{~Hz}, 2 \mathrm{H}), 3.28(\mathrm{dd}, J 12.7,6.6 \mathrm{~Hz}, 2 \mathrm{H}), 2.28(\mathrm{~s}, 6 \mathrm{H}), 2.26(\mathrm{~s}, 12 \mathrm{H}), 2.04-1.98(\mathrm{~m}, 2 \mathrm{H}), 1.96-1.88(\mathrm{~m}$, 2H), $1.04(\mathrm{~d}, J 6.6 \mathrm{~Hz}, 6 \mathrm{H}), 0.70(\mathrm{~d}, J 6.7 \mathrm{~Hz}, 6 \mathrm{H}) ;{ }^{13} \mathrm{C} \mathrm{NMR}\left(125 \mathrm{MHz}, \mathrm{CDCl}_{3}\right) \delta 169.16,154.83,139.75,135.27$, $129.79,128.52,69.83,62.17,39.85,29.68,27.04,21.07,19.93,18.96,18.88$; HR-ESIMS: $\mathrm{m} / z$ 549.3754 [M$\left.\mathrm{BF}_{4}\right]^{+}$(calcd for $\mathrm{C}_{34} \mathrm{H}_{49} \mathrm{~N}_{2} \mathrm{O}_{4}{ }^{+}, 549.3687$ ).

6h. 73\% yield; ${ }^{1} \mathrm{H}$ NMR $\left(500 \mathrm{MHz} \mathrm{CDCl}_{3}\right) \delta 8.92(\mathrm{~d}, J 8.7 \mathrm{~Hz}, 2 \mathrm{H}), 8.62(\mathrm{~s}, 1 \mathrm{H}), 8.38$ (dd, J 7.3, $\left.1.1 \mathrm{~Hz}, 2 \mathrm{H}\right), 8.03$ (d, J $8.2 \mathrm{~Hz}, 2 \mathrm{H}), 7.88(\mathrm{~d}, J 8.1 \mathrm{~Hz}, 2 \mathrm{H}), 7.64(\mathrm{~m}, 2 \mathrm{H}), 7.58-7.51(\mathrm{~m}, 4 \mathrm{H}), 4.75(\mathrm{dd}, J 12.5,10.4 \mathrm{~Hz}, 2 \mathrm{H}), 4.39$ (dd, J 12.5, $3.4 \mathrm{~Hz}, 2 \mathrm{H}), 4.00(\mathrm{~m}, 2 \mathrm{H}), 3.49-3.40(\mathrm{~m}, 2 \mathrm{H}), 3.16-3.09(\mathrm{~m}, 2 \mathrm{H}), 2.02-1.95(\mathrm{~m}, 2 \mathrm{H}), 1.83-1.74(\mathrm{~m}$, $2 \mathrm{H}), 1.00$ (d, J $6.6 \mathrm{~Hz}, 6 \mathrm{H}), 0.40(\mathrm{~d}, J 6.6 \mathrm{~Hz}, 6 \mathrm{H}) ;{ }^{13} \mathrm{C} \mathrm{NMR}\left(125 \mathrm{MHz}, \mathrm{CDCl}_{3}\right) \delta$ 166.64, 155.07, 134.12, 133.77, $131.43,128.68,128.11,126.27,125.39,125.11,124.97,124.54,70.28,61.16,39.07,29.68,19.03,18.88$; HRESIMS: $m / z 565.3186\left[\mathrm{M}-\mathrm{BF}_{4}\right]^{+}$(calcd for $\mathrm{C}_{36} \mathrm{H}_{41} \mathrm{~N}_{2} \mathrm{O}_{4}{ }^{+}, 565.3061$ ).

6i. $86 \%$ yield; ${ }^{1} \mathrm{H}$ NMR $\left(500 \mathrm{MHz}, \mathrm{CDCl}_{3}\right) \delta 8.73(\mathrm{~s}, 2 \mathrm{H}), 8.67(\mathrm{~s}, 1 \mathrm{H}), 8.08(\mathrm{~d}, J 8.0 \mathrm{~Hz}, 2 \mathrm{H}), 8.02(\mathrm{dd}, J 8.6,1.6 \mathrm{~Hz}$, $2 \mathrm{H}), 7.86(\mathrm{dd}, J 8.3,3.1 \mathrm{~Hz}, 4 \mathrm{H}), 7.64-7.54(\mathrm{~m}, 4 \mathrm{H}), 4.84(\mathrm{dd}, J 12.3,10.6 \mathrm{~Hz}, 2 \mathrm{H}), 4.30$ (dd, J 12.4, 3.4 Hz, 2H), $4.03(\mathrm{~m}, 2 \mathrm{H}), 3.50-3.39(\mathrm{~m}, 2 \mathrm{H}), 3.21-3.09(\mathrm{~m}, 2 \mathrm{H}), 2.05-1.97(\mathrm{~m}, 2 \mathrm{H}), 1.86-1.72(\mathrm{~m}, 2 \mathrm{H}), 0.96(\mathrm{~d}, \mathrm{~J} 6.6 \mathrm{~Hz}$, $6 \mathrm{H}), 0.42(\mathrm{~d}, J 6.6 \mathrm{~Hz}, 6 \mathrm{H}) ;{ }^{13} \mathrm{C}$ NMR $\left(125 \mathrm{MHz}, \mathrm{CDCl}_{3}\right) \delta 166.33,155.02,135.70,132.48,131.77,129.73,128.60$, $128.37,127.58,126.79,125.98,124.96,70.38,61.26,39.21,26.79,19.03,18.98 ;$ HR-ESIMS: m/z 565.3191 [M$\left.\mathrm{BF}_{4}\right]^{+}$(calcd for $\mathrm{C}_{36} \mathrm{H}_{41} \mathrm{~N}_{2} \mathrm{O}_{4}{ }^{+}, 565.3061$ ).

Representative procedure for the asymmetric addition of diethylzinc to aldehyde. Under argon atmosphere, a mixture of salt $6 \mathbf{b}(0.01 \mathrm{mmol})$ and KHMDS $(0.03 \mathrm{mmol})$ in xylene $(1 \mathrm{~mL})$ was stirred for 5 min at room temperature. Then diethylzinc $(0.2 \mathrm{mmol})$ was added dropwise, followed by addition of aldehdye 7 (0.1 $\mathrm{mmol})$. Upon stirring for $24 \mathrm{~h}$ at room temperature, the reaction was quenched by $\mathrm{HCl}(1 \mathrm{M}, 1.0 \mathrm{~mL})$, and extracted with $\mathrm{Et}_{2} \mathrm{O}(3 \times 2 \mathrm{~mL})$. The combined organic phases were washed with water and dried over $\mathrm{Na}_{2} \mathrm{SO}_{4}$ and concentrated under vacuum. The residue was further purified by column chromatography (silica gel, hexane/AcOEt) to give product 8.

8a. $93 \%$ yield, $58 \%$ ee; the spectral data were comparable to those reported. ${ }^{29}$ The ee was determined by HPLC analysis with Daicel Chiralcel OD-H (hexane/ ${ }^{i} \mathrm{PrOH}=90 / 10$, flow rate $=0.5 \mathrm{~mL} / \mathrm{min}, \mathrm{t}_{\mathrm{r}}(\operatorname{minor})=15.7 \mathrm{~min}$, $\mathrm{t}_{\mathrm{r}}$ (major) $\left.=28.6 \mathrm{~min}\right)$.

8b. $83 \%$ yield, $34 \%$ ee; The spectral data were comparable to those reported. ${ }^{30}$ The ee was determined by HPLC analysis with Daicel Chiralcel OD-H (hexane/ ${ }^{\mathrm{PrOH}}=90 / 10$, flow rate $=0.5 \mathrm{~mL} / \mathrm{min}, \mathrm{t}_{\mathrm{r}}$ (minor) $=19.2 \mathrm{~min}$, $t_{r}($ major $\left.)=22.5 \mathrm{~min}\right) .8 \mathrm{c} .91 \%$ yield, 37\% ee; The spectral data were comparable to those reported. ${ }^{31}$ The ee was determined by HPLC analysis with Daicel Chiralcel OD-H (hexane/i $\mathrm{PrOH}=90 / 10$, flow rate $=0.5 \mathrm{~mL} / \mathrm{min}, \mathrm{t}_{\mathrm{r}}$ (major) $=10.6 \mathrm{~min}, \mathrm{t}_{\mathrm{r}}$ (minor) $\left.=12.2 \mathrm{~min}\right) . \mathbf{8 d}$. 74\% yield, $43 \%$ ee; The spectral data were comparable to those reported. ${ }^{31}$ The ee was determined by HPLC analysis with Daicel Chiralcel OD-H (hexane/'PrOH $=90 / 10$, flow rate $=0.5 \mathrm{~mL} / \mathrm{min}, \mathrm{t}_{\mathrm{r}}$ (major) $=12.7 \mathrm{~min}, \mathrm{t}_{\mathrm{r}}$ (minor) $=15.4 \mathrm{~min}$ ). 8e. 79\% yield, 50\% ee; The spectral data were comparable to those reported. ${ }^{29}$ The ee was determined by HPLC analysis with Daicel Chiralcel OD-H (hexane/ $\mathrm{PrOH}=90 / 10$, flow rate $=0.5 \mathrm{~mL} / \mathrm{min}, \mathrm{t}_{\mathrm{r}}$ (minor) $=18.3 \mathrm{~min}, \mathrm{t}_{\mathrm{r}}$ (major) $=20.5 \mathrm{~min}$ ). 8 f. $92 \%$ yield, $20 \%$ ee; The spectral data were comparable to those reported. ${ }^{28}$ The ee was determined by HPLC analysis with 
Daicel Chiralcel OD-H (hexane/i $\mathrm{PrOH}=90 / 10$, flow rate $=0.4 \mathrm{~mL} / \mathrm{min}, \mathrm{t}_{\mathrm{r}}($ minor $)=8.9 \mathrm{~min}, \mathrm{t}_{\mathrm{r}}($ major $)=9.7$ $\min )$.

8g. 63\% yield, $24 \%$ ee; The spectral data were comparable to those reported. ${ }^{29}$ The ee was determined by HPLC analysis with Daicel Chiralcel OD-H (hexane/ ${ }^{\mathrm{PrOH}}=90 / 10$, flow rate $=0.5 \mathrm{~mL} / \mathrm{min}, \mathrm{t}_{\mathrm{r}}(\operatorname{minor})=18.5 \mathrm{~min}$, $\mathrm{t}_{\mathrm{r}}($ major $\left.)=21.9 \mathrm{~min}\right)$.

8h. $75 \%$ yield, $36 \%$ ee; The spectral data were comparable to those reported. ${ }^{30}$ The ee was determined by HPLC analysis with Daicel Chiralcel OD-H (hexane/ ${ }^{i} \mathrm{PrOH}=90 / 10$, flow rate $=0.5 \mathrm{~mL} / \mathrm{min}, \mathrm{t}_{\mathrm{r}}$ (major) $=10.6 \mathrm{~min}$, $\left.\mathrm{t}_{\mathrm{r}}(\operatorname{minor})=12.2 \mathrm{~min}\right)$.

8i. $84 \%$ yield, $35 \%$ ee; The spectral data were comparable to those reported. ${ }^{32}$ The ee was determined by HPLC analysis with Daicel Chiralcel OD-H (hexane/ ${ }^{i} \mathrm{PrOH}=90 / 10$, flow rate $=0.5 \mathrm{~mL} / \mathrm{min}, \mathrm{t}_{\mathrm{r}}$ (major) $=11.9 \mathrm{~min}$, $\mathrm{t}_{\mathrm{r}}$ (minor) $\left.=15.3 \mathrm{~min}\right)$.

8j. $80 \%$ yield, $45 \%$ ee; The spectral data were comparable to those reported. ${ }^{29}$ The ee was determined by HPLC analysis with Daicel Chiralcel OD-H (hexane $/{ }^{\mathrm{P} P O H}=93 / 7$, flow rate $=0.5 \mathrm{~mL} / \mathrm{min}, \mathrm{t}_{\mathrm{r}}$ (major) $=11.6 \mathrm{~min}, \mathrm{t}_{\mathrm{r}}$ (minor) $=13.6 \mathrm{~min}$ ).

8k. $90 \%$ yield, $36 \%$ ee; The spectral data were comparable to those reported. ${ }^{32}$ The ee was determined by HPLC analysis with Daicel Chiralcel OD-H (hexane $/{ }^{\mathrm{P} P O H}=93 / 7$, flow rate $=0.5 \mathrm{~mL} / \mathrm{min}, \mathrm{t}_{\mathrm{r}}(\operatorname{minor})=11.9 \mathrm{~min}, \mathrm{t}_{\mathrm{r}}$ (major) $=12.8 \mathrm{~min})$.

81. $83 \%$ yield, $28 \%$ ee; The spectral data were comparable to those reported. ${ }^{28}$ The ee was determined by HPLC analysis with Daicel Chiralcel OD-H (hexane/ ${ }^{i} \mathrm{PrOH}=93 / 7$, flow rate $=0.5 \mathrm{~mL} / \mathrm{min}, \mathrm{t}_{\mathrm{r}}(\operatorname{major})=11.2 \mathrm{~min}, \mathrm{t}_{\mathrm{r}}$ (minor) $=13.1 \mathrm{~min})$.

$8 \mathrm{~m}$. $87 \%$ yield, $60 \%$ ee; The spectral data were comparable to those reported. ${ }^{29}$ The ee was determined by HPLC analysis with Daicel Chiralcel OD-H (hexane/ $\mathrm{PrOH}=93 / 7$, flow rate $=0.5 \mathrm{~mL} / \mathrm{min}, \mathrm{t}_{\mathrm{r}}(\operatorname{minor})=14.1 \mathrm{~min}, \mathrm{t}_{\mathrm{r}}$ (major) $=15.8 \mathrm{~min}$ ).

8n. $73 \%$ yield, $45 \%$ ee; The spectral data were comparable to those reported. ${ }^{33}$ The ee was determined by HPLC analysis with Daicel Chiralcel OD-H (hexane $/ \mathrm{PrOH}=93 / 7$, flow rate $=0.5 \mathrm{~mL} / \mathrm{min}, \mathrm{t}_{\mathrm{r}}(\mathrm{minor})=13.6 \mathrm{~min}, \mathrm{t}_{\mathrm{r}}$ (major) $=14.6 \mathrm{~min}$ ).

80. $88 \%$ yield, $38 \%$ ee; The spectral data were comparable to those reported. ${ }^{30}$ The ee was determined by HPLC analysis with Daicel Chiralcel OD-H (hexane/ ${ }^{i} \mathrm{PrOH}=93 / 7$, flow rate $=0.5 \mathrm{~mL} / \mathrm{min}, \mathrm{t}_{\mathrm{r}}(\mathrm{major})=9.4 \mathrm{~min}, \mathrm{t}_{\mathrm{r}}$ $(\operatorname{minor})=10.7 \mathrm{~min})$.

8p. $76 \%$ yield, $66 \%$ ee; The spectral data were comparable to those reported. ${ }^{34}$ The ee was determined by HPLC analysis with Daicel Chiralcel OD-H (hexane/ ${ }^{2} \mathrm{PrOH}=90 / 10$, flow rate $=0.5 \mathrm{~mL} / \mathrm{min}, \mathrm{t}_{\mathrm{r}}(\operatorname{minor})=14.1 \mathrm{~min}$, $\mathrm{t}_{\mathrm{r}}$ (major) $=30.2 \mathrm{~min}$.

\section{Acknowledgements}

We are grateful to the National Natural Science Foundation of China (31670357) and Hangzhou Science and Technology Information Institute of China (20150633B45).

\section{Supplementary Material}

${ }^{1} \mathrm{H}$ and ${ }^{13} \mathrm{C}$ NMR spectra of new compounds and crystallographic data of $6 \mathbf{6 f}$. 


\section{References}

1. Arduengo, A. J. ; Harlow, R. L. ; Kline, M. J. Am. Chem. Soc. 1991, 113, 361-363.

https://doi.org/10.1021/ja00001a054

2. Herrmann, W. A. ; Köcher, C. Angew. Chem., Int. Ed. 1997, 36, 2162-2187.

https://doi.org/10.1002/anie.199721621

3. Bourissou, D.; Guerret, O.; Gabbaï, F.P.; Bertrand, G. Chem. Rev. 2000, 100, 39-92. https://doi.org/10.1021/cr940472u

4. Zinn, F. K.; Viciu, M. S.; Nolan, S. P. Annu. Rep. Prog. Chem. Sect B. 2004, 100, 231-249. https://doi.org/10.1039/B401751J

5. Cavell, K. J.; McGuinness, D. S. Coord. Chem. Rev. 2004, 248, 671-681.

https://doi.org/10.1016/j.ccr.2004.02.006

6. Peris, E.; Crabtree, R. H. Coord. Chem. Rev. 2004, 248, 2239-2246.

https://doi.org/10.1016/j.ccr.2004.04.014

7. Crudden, C. M.; Allen, D. P. Coord. Chem. Rev. 2004, 248, 2247-2273.

https://doi.org/10.1016/j.ccr.2004.05.013

8. Pugh, D.; Danopoulos, A. A. Coord. Chem. Rev. 2007, 251, 610-641.

https://doi.org/10.1016/j.ccr.2006.08.001

9. Mata, J. A.; Poyatos, M.; Peris, E. Coord. Chem. Rev. 2007, 251, 841-859.

https://doi.org/10.1016/j.ccr.2006.06.008

10. Sommer, W. J.; Weck, M. Coord. Chem. Rev. 2007, 251, 860-873.

https://doi.org/10.1016/j.ccr.2006.07.004

11. Hahn, F. E.; Jahnke, M. C. Angew. Chem., Int. Ed. 2008, 47, 3122-3172.

https://doi.org/10.1002/anie.200703883

12. Hopkinson, M.N.; Richter, C.; Schedler, M.; Glorius, F. Nature 2014, 510, 485-496.

https://doi.org/10.1038/nature13384

13. Perry, M. C.; Burgess, K. Tetrahedron: Asymmetry 2003, 14, 951-961.

https://doi.org/10.1016/S0957-4166(03)00037-5

14. César, V.; Bellemin-Laponnaz, S.; Gade, L. H. Chem. Soc. Rev. 2004, 33, 619-636. https://doi.org/10.1039/B406802P

15. Douthwaite, R. E. Coord. Chem. Rev. 2007, 251, 702-717.

https://doi.org/10.1016/j.ccr.2006.08.003

16. Gade, L. H.; Bellemin-Laponnaz, S. Coord. Chem. Rev. 2007, 251, 718-725.

https://doi.org/10.1016/i.ccr.2006.05.015

17. Snead, D. R.; Seo, H.; Hong, S. Curr. Org. Chem. 2008, 12, 1370-1387.

https://doi.org/10.2174/138527208786241510

18. Wang, F. J.; Liu, L. J.; Wang, W. F.; Li, S. K.; Shi, M. Coord. Chem. Rev. 2012, 256, 804-853. https://doi.org/10.1016/j.ccr.2011.11.013

19. Arnold, P. L.; Rodden, M.; Davis, K. M.; Scarisbrick, A. C.; Blake, A. J.; Wilson, C. Chem. Commun. 2004, 1612-1613.

https://doi.org/10.1039/b404614e

20. Clavier, H.; Boulanger, L.; Audic, N.; Toupet, L.; Mauduit, M.; Guillemin, J. C. Chem. Commun. 2004, 12241225. 
21. Clavier, H.; Coutable, L.; Guillemin, J. C.; Mauduit, M. Tetrahedron: Asymmetry 2005, 16, 921-924. https://doi.org/10.1016/j.tetasy.2005.01.015

22. Martin, D.; Kehrli, S.; d'Augustin, M.; Clavier, H.; Mauduit, M.; Alexakis, A. J. Am. Chem. Soc. 2006, 128, 8416-8417.

https://doi.org/10.1021/ja0629920

23. Magrez, M.; Guen, Y. L.; Baslé, Q.; Crévisy, C.; Mauduit, M. Chem. Eur. J. 2013, 19, 1199-1203. https://doi.org/10.1002/chem.201203969

24. Li, X. B.; Meng, F. K.; Torker, S. B.; Shi, Y. Hoveyda, A. H. Angew. Chem., Int. Ed. 2016, 55, 9997-10002. https://doi.org/10.1002/anie.201605001

25. Jurčík, V.; Gilani, M.; Wilhelm, R. Eur. J. Org. Chem. 2006, 5103-5109. https://doi.org/10.1002/ejoc.200600584

26. Gilani, M.; Wilhelm, R. Tetrahedron: Asymmetry 2008, 19, 2346-2352. https://doi.org/10.1016/i.tetasy.2008.10.011

27. Zhou, B. H.; Wu, C.; Chen, X. X.; Huang, H. X.; Li, L. L.; Fan, L. M.; Li, J. Tetrahedron Lett. 2017, 58, 41574161.

https://doi.org/10.1016/j.tetlet.2017.09.022

28. Zhou, B. H.; He, W. P.; Li, L. L.; Fan, L. M.; Li, X. R.; Li, J. Curr. Org. Syn. 2017, 14, DOI: 10.2174/1570179414666170830130658 https://doi.org/10.2174/1570179414666170830130658

29. Faigl, F.; Erdélyi, Z.; Deák, S.; Nyerges, M.; Mátravölgyi, B. Tetrahedron Lett. 2015, 46, 6891-6894.

30. Aga, M. A.; Kumar, B.; Rouf, A.; Shah, B. A.; Taneja, S. C. Tetrahedron Lett. 2014, 55, 2639-2641. https://doi.org/10.1016/j.tetlet.2014.03.002

31. Yue, H. F.; Huang, H. Y; Bian, G. L.; Zong, H.; Li, F. L.; Song, L. Tetrahedron: Asymmetry 2014, 25, $170-180$. https://doi.org/10.1016/i.tetasy.2013.12.014

32. Zhang, P. X.; Yu, J. P.; Peng, F.; Wu, X. D.; Jie, J. Y.; Liu, C.; Tian, H.; Yang, H. J.; Fu, H. Chem. Eur. J. 2016, 22, 17477-17484.

https://doi.org/10.1002/chem.201603706

33. Lougheed, S.C.; Campagna, L.; Dávila, J. A.; Tubaro, P. L.; Lijtmaer, D. A. Tetrahedron 1998, 29, 1031710328.

34. Fakhfakh, M. A.; Fournet, A.; Prina, E.; Mouscadet, J. F.; Franck, X.; Hocquemiller, R.; Figadèrea, B. Bioorg. Med. Chem. 2003, 11, 5013-5023.

https://doi.org/10.1016/j.bmc.2003.09.007 